\title{
Passivity-based control for stabilization, regulation and tracking purposes of a class of nonlinear systems
}

\author{
Juan C. Travieso-Torres, Manuel A. Duarte-Mermoud*,† and Diego I. Sepúleveda \\ Department of Electrical Engineering, University of Chile, Av.Tupper 2007, Casilla 412-3, Santiago, Chile
}

\begin{abstract}
SUMMARY
In this paper, a new passivity-based control (PBC) scheme based on state feedback is proposed in order to solve tracking, regulation and stabilization problems for a class of multi-input multi-output (MIMO) nonlinear systems expressed in the normal form, with time-invariant parameters and locally bounded reference weakly minimum phase. For the proposed control scheme two new different state feedbacks, one non-adaptive for the case when the system parameters are assumed to be known and the other adaptive for the case of unknown parameters, are developed. For the adaptive case it is assumed that the unknown parameters appear linearly in the equations. Analysis of the transient behaviour of the proposed control schemes is presented through the simulation of two examples. Copyright (C) 2006 John Wiley \& Sons, Ltd.
\end{abstract}

Received 1 April 2005; Revised 3 August 2006; Accepted 25 September 2006

KEY WORDS: passivity-based control; adaptive passivity; adaptive tracking; adaptive regulation; adaptive stabilization; adaptive nonlinear control

\section{INTRODUCTION}

During the last three decades, feedback stabilization of nonlinear systems has been widely studied [1-13]. Different techniques, non-adaptive as well as adaptive, have been used to solve this problem. Amongst the most interesting we can cite is the work by Aeyels in 1985 [1] which considers the stabilization problem via non-adaptive smooth feedback for systems of the form $\dot{x}(t)=f(x)+b u(t)$ in the neighbourhood of an equilibrium point. Additionally, by means of centre manifold theory a lower order system is introduced, showing that if this system is stabilizable then also is the original system. Marino and Tomei [6] presented a global robust stabilizing non-adaptive state feedback controller for a class of single-input nonlinear systems.

\footnotetext{
*Correspondence to: Manuel A. Duarte-Mermoud, Department of Electrical Engineering, University of Chile, Av.Tupper 2007, Casilla 412-3, Santiago, Chile.

†E-mail: mduartem@cec.uchile.cl

Contract/grant sponsor: CONICYT-CHILE; contract/grant number: 1030962
} 
The system has bounded unmodelled time-varying disturbances, whose bounds are known, entering nonlinearly in the state equations. The design assumes that the undisturbed system is globally feedback linearizable and that a triangularity condition holds for the uncertain terms.

For the case of stabilizing locally weakly minimum-phase nonlinear systems with relative degree 1, passivity-based control (PBC) is an important methodology [2-5, 8-10]. This technique is based on two steps; first, it obtains a $C^{r}$-passive equivalent nonlinear system via state feedback, and then a stabilizing controller is applied to the passive system, which is easier to control than the original system. If the system is already passive the controller can be directly applied. A synthesis of concepts and conditions under which the nonlinear system with zero equilibrium point, represented in the normal form, can be rendered $C^{r}$-passive via smooth nonadaptive state feedback are presented in [5]. The system considered has the following form:

$$
\begin{aligned}
\dot{y}(t) & =a(A, y, z)+b(B, y, z) u(t) \\
\dot{z}(t) & =f_{0}(C, z)+p(P, y, z) y(t)
\end{aligned}
$$

where $f_{0}(C, z)$ is the zero dynamics and all the system parameters are assumed to be known.

Feedback passivity by means of state feedback for systems containing uncertain elements is studied in [7-10]. This problem is also treated in [14-17] for nonlinear systems with linear parametric uncertainties. Here, adaptive state feedbacks to obtain $C^{r}$-passive equivalent systems were proposed for nonlinear systems considering the same conditions as in [5], but restricted to a class of nonlinear systems with linear explicit parametric dependence in the normal form expressed as

$$
\begin{aligned}
& \dot{y}(t)=A a^{\prime}(y, z)+B b^{\prime}(y, z) u(t) \\
& \dot{z}(t)=C f_{0}^{\prime}(z)+p^{\prime}(y, z) P y(t)
\end{aligned}
$$

where $A, B, C$ and $P$ are unknown parameters. These adaptive techniques assume unknown parameters and they are robust under parameter variations. For the case where all parameters of this reduced class of nonlinear systems with linear explicit parametric dependence are assumed to be known, the non-adaptive solution is a particularization of the adaptive solution. The results from [14,17] are developed for stabilizing single-input single-output (SISO) nonlinear systems, using time-varying adaptive gains and also for fixed adaptive gains for adjusting the adaptive parameters, respectively. The results from $[15,16]$ are extensions for the stabilization of multi-input multi-output (MIMO) nonlinear systems. The stabilization scheme shown in Figure 1 has been proposed by Byrnes et al. [5], Duarte-Mermoud et al. [14-16] and

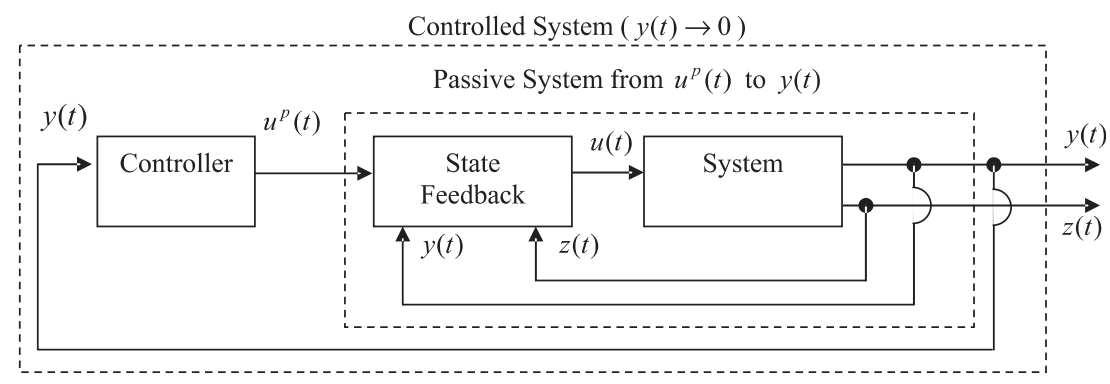

Figure 1. General PBC scheme for stabilization purposes. 
Castro-Linares and Duarte-Mermoud [17] for the non-adaptive and adaptive cases. Once the system is rendered $C^{r}$ passive, if it is locally zero-state observable, a controller of the form $u^{p}(t)=-K y(t)$ with $K \in \mathfrak{R}^{m \times m}$ any positive definite matrix, asymptotically stabilizes the system around the equilibrium point $x=0$.

The equivalence between model reference adaptive control (MRAC) theory [18] and the adaptive PBC for stabilization purposes [17] has been established in [19]. In [18, 19] it is shown that obtaining an asymptotically stable error model (by any technique) is the main objective of the adaptive control theory. Based on the results given in [19] the adaptive state feedback presented in this paper was developed.

A generalization of the nonlinear non-adaptive [5] and adaptive state feedback [17] proposed earlier for stabilization is presented in this paper. The new state feedbacks are proposed as part of a new control scheme, which also considers a controller for tracking an arbitrary differentiable reference signal, this being the main result of this paper. In this case locally bounded reference weakly minimum-phase property is needed to achieve tracking.

For completeness, in Section 2 of this paper, the basic concepts of non-adaptive and adaptive state feedbacks involved in a PBC scheme for stabilization of nonlinear systems are briefly exposed. In Section 3, a new general PBC scheme for tracking purposes, together with nonadaptive and adaptive state feedbacks, is proposed. Simulation results of the proposed control schemes for the non-adaptive and adaptive cases are exposed in Section 4, to verify the behaviour of the controlled system. Finally, in Section 5 some conclusions are drawn.

\section{PBC RELATED CONCEPTS}

Basic passivity concepts given in [5] consider a nonlinear system of the form

$$
\begin{aligned}
& \dot{x}(t)=f(x)+g(x) u(t) \\
& y(t)=h(x)
\end{aligned}
$$

with state space $X=\mathfrak{R}^{n}$, set of input values on $U=\mathfrak{R}^{m}$ and set of output values $Y=\mathfrak{R}^{m}$. The set $U$ of admissible inputs consist of all piecewise continuous functions $\mathfrak{R} \rightarrow U=\mathfrak{R}^{m}$. Besides, $f \in \mathfrak{R}^{n}$ and $g \in \mathfrak{R}^{n \times m} . \quad f$ and the $m$ columns of $g$ are smooth vector fields (i.e. $f, g \in C^{\infty}$ ), and $h \in \mathfrak{R}^{m}$ is a smooth mapping $\left(h \in C^{\infty}\right)$. It is supposed that the vector field $f$ has at least one equilibrium point and without loss of generality, after possibly a co-ordinate shift, we can write that $f(0)=0$ and $h(0)=0$ [5]. Next, for completeness we recall some definitions and assumptions from [5], particularized for systems of the form (1).

Definition 2.1 (Byrnes et al. [5])

A system of the form (1) is said to be $C^{r}$-passive if there exists a $C^{r}$ non-negative function $V: \mathfrak{R}^{n} \rightarrow \mathfrak{R}$, called storage function, with $V(0)=0$, such that for all $u \in U$, for all $x(0)=x_{0} \in X$ and $t \geqslant 0$, it satisfies

$$
V(x(t))-V\left(x_{0}\right) \leqslant \int_{0}^{t} y^{\mathrm{T}}(s) u(s) \mathrm{d} s
$$

where $V$ is a continuous storage function with continuous $r$-order derivatives $\left(V \in C^{r}\right)$. Condition (2) can also be expressed as $\dot{V} \leqslant y(t)^{\mathrm{T}} u(t)$. The system is said to be lossless for the case when $\dot{V}=y(t)^{\mathrm{T}} u(t)$. 
Definition 2.2 (Byrnes et al. [5])

A system of the form (1) is locally zero-state detectable if there exists a neighbourhood $N$ of 0 such that for all $x_{0} \in N$ we have

$$
h\left(\Phi\left(t, x_{0}, 0\right)\right)=0 \quad \text { for all } t \geqslant 0 \Rightarrow \lim _{t \rightarrow \infty} \Phi\left(t, x_{0}, 0\right)=0
$$

where $\Phi\left(t, x_{0}, 0\right)$ denotes the state response at time $t$ for zero input, starting from the initial state $x_{0}$ at $t=0$. If $N=X$, then the system is said to be zero-state detectable.

System (1) is locally zero-state observable if there exists a neighbourhood $N$ of 0 such that for all $x_{0} \in N$ we have

$$
h\left(\Phi\left(t, x_{0}, 0\right)\right)=0 \quad \text { for all } t \geqslant 0 \Rightarrow x=0
$$

If $N=X$, then the system is said to be zero-state observable.

Assumption 2.1 (Byrnes et al. [5])

Let us assume that system (1) has relative degree $\{1,1,1, \ldots, 1\}$ at $x=0$, the matrix $L_{g} h(x)=(\partial h(x) / \partial x) g(x)$ is non-singular in a neighbourhood of $x=0$, and the distribution spanned by vector fields $g_{1}(x), \ldots, g_{m}(x)$ is involutive.

Definition 2.3 (Byrnes et al. [5])

It is shown in [5] that for a system (1) under Assumption 2.1, it is possible to find a new set of local co-ordinates $z(x) \in \mathfrak{R}^{n-m}, m \leqslant n$, defined around $x=0$ and vanishing at $x=0$, under which this system, together with the $m$ components of the output map $y=h(x)$, can be represented in the normal form as follows:

$$
\begin{aligned}
\dot{y}(t) & =a(A, y, z)+b(B, y, z) u(t) \\
\dot{z}(t) & =c(C, y, z)
\end{aligned}
$$

where $a(A, y, z) \in \mathfrak{R} m, b(B, y, z) \in \mathfrak{R}^{m \times m}, c(C, y, z) \in \mathfrak{R}^{n-m}$ and $b(B, y, z)$ is invertible for all $(y, z)$ around $(0,0)$. The zero dynamics of system (4), denoted as $\dot{z}=c(C, 0, z)=f_{0}(C, z) \in \mathfrak{R}^{n-m}$, is defined [5] as those internal dynamics which are consistent with the external constraint $y=0$. Thus, system (4) can be represented as

$$
\begin{aligned}
& \dot{y}(t)=a(A, y, z)+b(B, y, z) u(t) \\
& \dot{z}(t)=f_{0}(C, z)+p(P, y, z) y(t)
\end{aligned}
$$

where $f_{0}(C, z) \in \mathfrak{R}^{n-m}$ and $p(P, y, z) \in \mathfrak{R}^{(n-m) \times m} . A, B, C$ and $P$ are symbolic representations of the system parameters. Note that $c(C, y, z)=f_{0}(C, z)+p(P, y, z) y(t) \in \mathfrak{R}^{n-m}$.

Definition 2.4 (Byrnes et al. [5])

Let us assume that $L_{g} h(0)$ is non-singular. System (5) is said to be locally weakly minimum phase if there exists a positive definite $C^{r}$-function $W(z)$, defined near 0 , with $r \geqslant 2$ and $W(0)=0$, satisfying

$$
L_{f_{0}(C, z)} W(z)=\frac{\partial W(z)}{\partial z} f_{0}(C, z) \leqslant 0
$$

for all $C$ and all $z$ in the neighbourhood of $z(t)=0$. 
Definition 2.5 (Byrnes et al. [5])

System (1) is said to be locally equivalent via feedback to a $C^{r}$-passive system, if there exists a state feedback $u(t)$ such that the resulting closed-loop system is passive with a proper $C^{r}$-storage function $V$, according to Definition 2.1.

Assumption 2.2 (Byrnes et al. [5])

Let us assume that system (2) is locally weakly minimum phase, according to Definition 2.4.

Assumption 2.3 (Byrnes et al. [5])

Let us assume that system (4) has a matrix $b(B, y, z)$ which is invertible for all $(y, z)$ (globally invertible).

For a system of form (1) under Assumptions 2.1-2.3 the following stabilization scheme shown in Figure 1 has been proposed [5,14-17] for the non-adaptive and adaptive cases.

\subsection{Non-adaptive stabilization using $P B C$}

Let us consider system (1) under Assumptions 2.1-2.3. We will first assume that plant parameters and vector fields of its normal form (5) are completely known. To make system (5) equivalent via feedback to a $C^{r}$-passive system, in [5] it is chosen a state feedback of the form

$$
u(t)=b(B, y, z)^{-1} \theta \omega(t)
$$

where $\quad \theta=\left[\begin{array}{lll}-I_{m} & -I_{m} & I_{m}\end{array}\right] \in \mathfrak{R}^{m \times 3 m}, \quad \omega=\left[\begin{array}{lll}a^{\mathrm{T}}(A, y, z) & \left(L_{p(P, y, z)} W_{0}(z)\right)^{\mathrm{T}}\left(u^{p}(t)\right)^{\mathrm{T}}\end{array}\right]^{\mathrm{T}} \in \mathfrak{R}^{3 m} \quad$ and $u^{p}(t) \in \mathfrak{R}^{m}$ is a new control input. $b(B, y, z) \in \mathfrak{R}^{m \times m}, a(A, y, z) \in \mathfrak{R}^{m}$ and $L_{p(P, y, z)} W_{0}(z) \in \mathfrak{R}^{m}$ are smooth functions defined locally near $x=0$ and $b(B, y, z)$ are invertible for all $(y, z)$ around $(0,0)$, (Assumption 2.3). $I_{m}$ denotes the identity matrix of dimension $m$. For the resultant closed-loop system we can write

$$
\begin{aligned}
\dot{V}(y, z)= & \left(\frac{\partial V(y, z)}{\partial y}\right)^{\mathrm{T}}\left[a(A, y, z)+b(B, y, z) b(B, y, z)^{-1} \theta \omega(t)\right] \\
& +L_{f_{0}(C, z)} W_{0}(z)+\left(L_{p(P, y, z)} W_{0}(z)\right)^{\mathrm{T}} y
\end{aligned}
$$

where $V$ is a positive $C^{r}$-storage function defined as $V(y, z)=(1 / 2) y^{\mathrm{T}} y+W_{0}(z)$, with $W_{0}(z)$ satisfying (6) and $V(0)=0$. Since $\partial V(y, z) / \partial y=y$, we will have the following:

$$
\dot{V}(y, z)=y^{\mathrm{T}}[a(A, y, z)+\theta \omega(t)]+L_{f_{0}(C, z)} W_{0}(z)+\left(L_{p(P, y, z)} W_{0}(z)\right)^{\mathrm{T}} y
$$

Replacing $\theta$ and $\omega$ in (7) we get

$$
\dot{V}(y, z)=y^{\mathrm{T}}(t) u^{p}(t)+L_{f_{0}(C, z)} W_{0}(z) \leqslant y^{\mathrm{T}}(t) u^{p}(t)
$$

where in the previous expression we have used Assumption 2.2.

According to Definition 2.5, the system has been turned passive from $u^{p}(t)$ to $y(t)$ via the state feedback (7) and if it is zero-state observable, according to Definition 2.2, then this system can be stabilized using the results contained in Theorem 2.1 stated next.

Theorem 2.1 (Byrnes et al. [5])

Let us consider a passive system of the form (5) satisfying Assumptions 2.1-2.3, with a $C^{r},(r \geqslant 1)$ proper storage function $V$, which is positive definite. Suppose the system is locally zero-state 
observable, according to Definition 2.2. Then the controller

$$
u^{p}(t)=-K y(t)
$$

with $K \in \mathfrak{R}^{m \times m}$ any positive definite matrix, asymptotically stabilizes the system around the equilibrium point $x=0$.

Proof

Replacing the control law given by (9) in inequality (8), we can obtain the following:

$$
\dot{V}(y, z) \leqslant-y(t)^{\mathrm{T}} K y(t)
$$

which is negative semi-definite, assuring stability of the equilibrium $x=0$, and therefore $y, z \in$ $l^{\infty}$. Integrating both sides of Equation (10) we obtain

$$
\int_{0}^{\infty} \dot{V} \mathrm{~d} t \leqslant-\int_{0}^{\infty} y(t)^{\mathrm{T}} K y(t) \mathrm{d} t
$$

or equivalently

$$
V(\infty)-V(0) \leqslant-\int_{0}^{\infty} y(t)^{\mathrm{T}} K y(t) \mathrm{d} t
$$

Therefore, we can conclude that $y(t) \in l^{2}$. From the controller definition given in (7) and since $u^{p}(t) \in l^{\infty}$, we conclude that $u(t) \in l^{\infty}$. From (5) we can write $\dot{y} \in l^{\infty}$. Using the Barbalat Lemma [18], since $y(t) \in l^{2}$ and $\dot{y}(t) \in l^{\infty}$, then $\lim _{t \rightarrow \infty} y(t)=0$. Since the system is locally zero-state observable and additionally $\lim _{t \rightarrow \infty} y(t)=0$ then we can conclude that $\lim _{t \rightarrow \infty} z(t)=0$.

From the above result we can conclude that the system is asymptotically stable around the equilibrium point $x=0$. The resulting PBC scheme is shown in Figure 1, where the state feedback is fixed, since the parameters are assumed to be known.

If system (1) is zero-state observable and $V$ is proper, controller (9) globally asymptotically stabilizes the equilibrium point $x=0$.

\subsection{Adaptive stabilization using $P B C$}

Let us consider system (1) under Assumptions 2.1-2.3, unknown and constant plant parameters, and with linear explicit parametric dependence of the form

$$
\begin{aligned}
& \dot{y}(t)=A a^{\prime}(y, z)+B b^{\prime}(y, z) u(t) \\
& \dot{z}(t)=f_{0}(C, z)+p^{\prime}(y, z) P y(t)
\end{aligned}
$$

where $a^{\prime}(y, z) \in \mathfrak{R}^{p}, b^{\prime}(y, z) \in \mathfrak{R}^{m \times m}, f_{0}(C, z) \in \mathfrak{R}^{n-m}, p^{\prime}(y, z) \in \mathfrak{R}^{(n-m) \times m}$ and $b^{\prime}(y, z)$ are invertible for all $(y, z)$ (Assumption 2.4). $A \in \mathfrak{R}^{m \times p}, B \in \mathfrak{R}^{m \times m}, P \in \mathfrak{R}^{m \times m}$ are the parameters of the plant, and $B$ is invertible (Assumption 2.4). For system (11), under the new Assumption 2.4 stated below, we choose the following adaptive state feedback $[5,16]$

$$
u(t)=b^{\prime}(y, z)^{-1} \theta(t) \omega(t)
$$

where $\omega=\left[a^{\prime}(y, z)^{\mathrm{T}}\left(L_{p^{\prime}(y, z)} W_{0}(z)\right)^{\mathrm{T}} u^{p}(t)^{\mathrm{T}}\right]^{\mathrm{T}} \in \mathfrak{R}^{p+2 m} \cdot u^{p}(t) \in \mathfrak{R}^{m}$ is the new control input and $\theta(t)=\left[\begin{array}{lll}\theta_{1}(t) & \theta_{2}(t) & \theta_{3}(t)\end{array}\right] \in \mathfrak{R}^{m \times(p+2 m)}$ are adjustable parameters with $\theta_{1}(t) \in \mathfrak{R}^{m \times p}, \theta_{2}(t) \in \mathfrak{R}^{m \times m}$ 
and $\theta_{3}(t) \in \mathfrak{R}^{m \times m}$. These parameters are updated using the adaptive law

$$
\dot{\theta}(t)=-\operatorname{sign}(B) y(t) \omega(t)^{\mathrm{T}}
$$

In the above expression, matrix $B$ has been assumed to be diagonal and $\operatorname{sign}(B)$ represents a diagonal matrix in whose diagonal are located the sign of the elements of the diagonal matrix $B$, which are assumed to be known (see Remark 2.1 for more general forms of $B$ ).

Assumption 2.4 (Castro-Linares and Duarte-Mermoud [17])

Let us assume that $B$ is a diagonal matrix and that matrices $B$ and $b^{\prime}(y, z)$ are invertible for all $(y, z)$ (globally invertible).

Let us consider the storage function $V(y, z, \Phi)=\frac{1}{2} y^{\mathrm{T}} y+W_{0}(z)+\frac{1}{2} \operatorname{Trace}\left(|B| \Phi^{\mathrm{T}} \Phi\right)$. For the resultant closed-loop system the following equality is satisfied

$$
\begin{aligned}
\dot{V}(y, z, \Phi)= & \left(\frac{\partial V(y)}{\partial y}\right)^{\mathrm{T}}\left[A a^{\prime}(y, z)+B b^{\prime}(y, z) b^{\prime}(y, z)^{-1} \theta(t) \omega(t)\right] \\
& +L_{f_{0}(C, z)} W_{0}(z)+\left(L_{p^{\prime}(y, z)} W_{0}(z)\right)^{\mathrm{T}} P y+\operatorname{Trace}\left(|B| \dot{\Phi}(t)^{\mathrm{T}} \Phi(t)\right)
\end{aligned}
$$

where $\Phi(t)=\theta(t)-\theta^{*} \in \mathfrak{R}^{m \times(p+2 m)}$ is the parameter error with the ideal parameters $\theta^{*}=\left[\begin{array}{lll}\theta_{1}^{*} & \theta_{2}^{*} & \theta_{3}^{*}\end{array}\right]=B^{-1}\left[\begin{array}{lll}-A & -P & I\end{array}\right] \in \mathfrak{R}^{m \times(p+2 m)} . V$ is a $C^{r}$-storage function with $V(0)=0 .|B|$ represents a diagonal matrix in whose diagonal are located the magnitude of the elements of the diagonal matrix $B$, which are assumed to be unknown. Since $\partial V(y, z, \Phi) / \partial y=y$, we can write the following:

$$
\begin{aligned}
\dot{V}(y, z, \Phi)= & y^{\mathrm{T}}\left[A a^{\prime}(y, z)+B \theta(t)^{\mathrm{T}} \omega(t)\right] \\
& +L_{f_{0}(C, z)} W_{0}(z)+\left(L_{p^{\prime}(y, z)} W_{0}(z)\right)^{\mathrm{T}} P y+\operatorname{Trace}\left(|B| \dot{\Phi}(t)^{\mathrm{T}} \Phi(t)\right)
\end{aligned}
$$

Replacing $\theta$ and $\omega$ in (12) we have

$$
\begin{aligned}
\dot{V}(y, z, \Phi)= & y^{\mathrm{T}}\left[A a^{\prime}(y, z)+B \theta_{1}(t) a^{\prime}(y, z)+B \theta_{2}(t) L_{p^{\prime}(y, z)} W_{0}(z)+B \theta_{3}(t) u^{p}(t)-u^{p}(t)\right] \\
& +y^{\mathrm{T}} u^{p}(t)+L_{f_{0}(C, z)} W_{0}(z)+\left(L_{p^{\prime}(y, z)} W_{0}(z)\right)^{\mathrm{T}} P y+\operatorname{Trace}\left(|B| \dot{\Phi}(t)^{\mathrm{T}} \Phi(t)\right)
\end{aligned}
$$

Since $\theta^{*}$ is constant then we replace $\dot{\Phi}(t)=\dot{\theta}(t)$ given by (13) to obtain the following:

$$
\dot{V}(y, z, \Phi)=y^{\mathrm{T}}(t) u^{p}(t)+L_{f_{0}(C, z)} W_{0}(z)
$$

From Definition 2.4 and Assumption 2.2, $L_{f_{0}(C, z)} W_{0}(z) \leqslant 0$ and we have

$$
\dot{V}(y, z, \Phi) \leqslant y^{\mathrm{T}}(t) u^{p}(t)
$$

According to Definition 2.5, the original system is equivalent via feedback to a passive one.

Furthermore, if the resulting system is zero-state observable according to Definition 2.2, then, the controller given in (9) asymptotically stabilizes the system around the equilibrium point $x=0$. The proof is similar to that given for Theorem 2.1 in Section 2.1 and therefore it is omitted. The reader is referred to $[15,16]$ for more details on the proof. The resulting adaptive PBC scheme is shown in Figure 1, where the state feedback block is now adaptive.

\section{Remark 2.1}

The solution given in (12) and (13) corresponds to the case when matrix $B$ is diagonal. Solutions for the cases, when $B$ is positive definite and for $B$ general, can be found in $[15,16]$. 
Remark 2.2

For the class of nonlinear system (11) studied, with linear explicit parametric dependence, the adaptive solution is a generalization of the non-adaptive one. The particular solution for the non-adaptive case considers a static state feedback with the controller parameter known, fixed

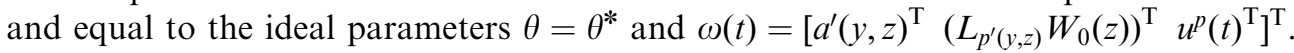

\section{PBC FOR TRACKING PURPOSES}

In this section a PBC scheme, which is valid for tracking purposes, is discussed. In what follows it will be assumed that the reference signal $y^{*}(t)$ and its first time derivative $\dot{y}^{*}(t)$ are available to the designer (Assumption 3.1). It is studied that a class of nonlinear systems (1), satisfying Assumptions 2.1-2.3 (or 2.4), which according to Definition 2.3, can be represented as

$$
\begin{aligned}
\dot{\tilde{y}}(t) & =a\left(A, \tilde{y}+y^{*}, z\right)+b\left(B, \tilde{y}+y^{*}, z\right) u(t)-\dot{y}^{*} \\
\dot{z}(t) & =c\left(C, \tilde{y}+y^{*}, z\right)
\end{aligned}
$$

where $\tilde{y}(t)=y(t)-y(t)^{*} . A, B$ and $C$ are symbolic representations of the system parameters. The zero dynamics of system (15), can be identified as $\dot{z}=c\left(C, 0+y^{*}, z\right)=f_{0}\left(C, y^{*}, z\right) \in \mathfrak{R}^{n-m}$. Thus, system (15) can be represented as

$$
\begin{aligned}
\dot{\tilde{y}}(t) & =a\left(A, \tilde{y}+y^{*}, z\right)+b\left(B, \tilde{y}+y^{*}, z\right) u(t)-\dot{y}^{*} \\
\dot{z}(t) & =f_{0}\left(C, y^{*}, z\right)+d\left(D, \tilde{y}+y^{*}, z\right) \tilde{y}
\end{aligned}
$$

where $d\left(D, \tilde{y}+y^{*}, z\right) \in \mathfrak{R}^{(n-m) \times m}$. Similar to the robust minimum-phase definition given in [7] we introduce the following definition.

\section{Definition 3.1}

System (16) is said to be locally bounded reference weakly minimum phase, if there exists a positive definite $C^{r}$-function $W_{0}(z)$, defined near $z=0$, with $r \geqslant 2$ and $W_{0}(0)=0$, satisfying

$$
L_{f_{0}\left(C, y^{*}, z\right)} W_{0}(z)=\frac{\partial W_{0}(z)}{\partial z} f_{0}\left(C, y^{*}, z\right) \leqslant 0
$$

for all $z$ in the neighbourhood of $z=0$, all bounded reference signals $y^{*}(t)$, and all values of parameters $C$.

Now we will assume that system (16) satisfies the following assumptions.

\section{Assumption 3.1}

Let us assume that system reference signal $y^{*}(t)$ is bounded and its time derivative exists and it is also bounded and known.

\section{Assumption 3.2}

Let us assume that system (16) is locally bounded reference weakly minimum phase, in the sense of Definition 3.1.

For systems of the form (16) under the Assumptions 3.1, 3.2 and 2.3 (or 2.4) the following general PBC scheme shown in Figure 2 is proposed. It is valid for the non-adaptive and the 


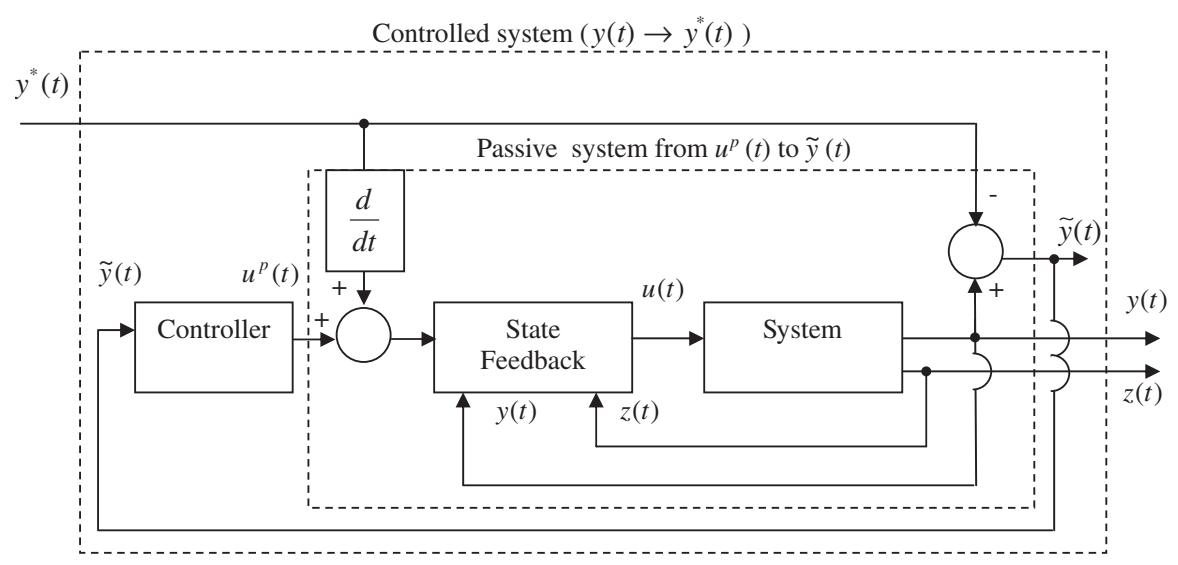

Figure 2. General PBC scheme for tracking purposes. Non-adaptive and adaptive cases.

adaptive cases by suitably choosing the state feedback. This scheme is a generalization of the schemes proposed earlier in [5,14-17] and presented in Figure 1.

\subsection{Non-adaptive PBC scheme}

If we assume that the parameters $A, B, C$ and $D$ are completely known, then we can state the following theorem regarding the tracking problem for systems that can be represented as in (16).

\section{Theorem 3.1}

Let us consider the system defined in (16) and suppose that Assumptions 3.1, 3.2 and 2.3 are satisfied, where $a(A, y, z) \in \mathfrak{R}^{m}, b(B, y, z) \in \mathfrak{R}^{m \times m}, d(D, y, z) \in \mathfrak{R}^{(n-m) \times m}$ and $f_{0}(C, y, z) \in \mathfrak{R}^{n-m}$. $A, B, C$ and $D$ represent the system parameters, which are assumed to be known. The following non-adaptive state feedback

$$
u(t)=b(B, y, z)^{-1} \theta \omega(t)
$$

with $\theta=\left[\begin{array}{ll}-I_{m} & I_{m}\end{array}\right] \in \mathfrak{R}^{m \times 2 m}$ and $\omega(t)=\left[a(A, y, z)^{\mathrm{T}}\left(u^{p}(t)-L_{d\left(D, \tilde{y}+y^{*}, z\right)} W_{0}(z)+\dot{y}^{*}(t)\right)^{\mathrm{T}}\right]^{\mathrm{T}} \in \mathfrak{R}^{2 m}$ makes system (16) equivalent to a passive system with a $C^{2}$-storage function. Furthermore, if we suppose that the system is locally zero-state observable, then applying the controller

$$
u^{p}(t)=-K \tilde{y}(t)
$$

with $K \in \mathfrak{R}^{m \times m}$ any positive definite matrix and $\tilde{y}(t)=y(t)-y(t)^{*} \in \mathfrak{R}^{m}$, the resulting overall system has bounded trajectories and $\lim _{t \rightarrow \infty} \tilde{y}(t)=y(t)-y(t)^{*}=0$.

Proof

After applying the state feedback (18) to system (16), it is as follows:

$$
\begin{aligned}
& \dot{\tilde{y}}(t)=a\left(A, \tilde{y}+y^{*}, z\right)+\theta \omega(t)-\dot{y}^{*}(t) \\
& \dot{z}(t)=f_{0}\left(C, y^{*}, z\right)+d\left(D, \tilde{y}+y^{*}, z\right) \tilde{y}
\end{aligned}
$$


and considering the definition of $\theta$ and $\omega(t)$ given in (18) it can be written as

$$
\begin{aligned}
& \dot{\tilde{y}}(t)=u^{p}(t)-L_{d\left(D, \tilde{y}+y^{*}, z\right)} W_{0}(z) \\
& \dot{z}(t)=f_{0}\left(C, y^{*}, z\right)+d\left(D, \tilde{y}+y^{*}, z\right) \tilde{y}
\end{aligned}
$$

Let us consider the positive definite storage function defined as $V(\tilde{y}, z)=\frac{1}{2} \tilde{y}(t)^{\mathrm{T}} \tilde{y}(t)+W_{0}(z)$. The first time derivative is

$$
\dot{V}(\tilde{y}, z)=\tilde{y}^{\mathrm{T}}(t) \dot{\tilde{y}}(t)+\dot{W}_{0}(z)
$$

Substituting $\dot{\tilde{y}}(t)$ from Equation (20) in the previous expression we obtain

$$
\dot{V}(\tilde{y}, z)=\tilde{y}^{\mathrm{T}}(t) u^{p}(t)-\tilde{y}^{\mathrm{T}}(t) L_{d\left(D, \tilde{y}+y^{*}, z\right)} W_{0}(z)+\left(\frac{\partial W_{0}(z)}{\partial z}\right)^{\mathrm{T}} \dot{z}(t)
$$

Replacing $\dot{z}(t)$ from Equation (20) in the previous expression and using Assumption 3.1 we get

$$
\begin{aligned}
\dot{V}(\tilde{y}, z)= & \tilde{y}^{\mathrm{T}}(t) u^{p}(t)-\tilde{y}^{\mathrm{T}}(t) L_{d\left(D, \tilde{y}+y^{*}, z\right)} W_{0}(z) \\
& +\left(\frac{\partial W_{0}(z)}{\partial z}\right)^{\mathrm{T}} f_{0}\left(C, y^{*}, z\right)+\left(\frac{\partial W_{0}(z)}{\partial z}\right)^{\mathrm{T}} d\left(D, \tilde{y}+y^{*}, z\right) \tilde{y} \\
= & \tilde{y}(t)^{\mathrm{T}} u^{p}(t)+L_{f_{0}\left(C, y^{*}, z\right)} W_{0}(z) \\
\leqslant & \tilde{y}(t)^{\mathrm{T}} u^{p}(t)
\end{aligned}
$$

In the previous expression we have used Assumption 3.1. Therefore, from Definition 2.5, we can conclude that the resultant system is passive from $u^{p}(t)$ to $\tilde{y}(t)$ with a $C^{2}$ storage function $V(\tilde{y}, z)$.

Replacing the controller $u^{p}(t)=-K \tilde{y}(t)$ in Equation (23) we get

$$
\dot{V}(\tilde{y}, z) \leqslant-\tilde{y}^{\mathrm{T}}(t) K \tilde{y}(t)
$$

which is negative semi-definite, assuring global boundedness of all trajectories of the adaptive system.

In particular, we can conclude that $\tilde{y}, z \in l^{\infty}$ and since $y^{*} \in l^{\infty}$ by hypothesis, then $y \in l^{\infty}$. Integrating both sides of Equation (24) we obtain the following:

$$
\int_{0}^{\infty} \dot{V} \mathrm{~d} t \leqslant-\int_{0}^{\infty} \tilde{y}(t)^{\mathrm{T}} K \tilde{y}(t) \mathrm{d} t
$$

or equivalently

$$
V(\infty)-V(0) \leqslant-\int_{0}^{\infty} \tilde{y}(t)^{\mathrm{T}} K \tilde{y}(t) \mathrm{d} t
$$

Therefore, we can conclude that $\tilde{y}(t) \in l^{2}$. From Equation (19) $u^{p}(t) \in l^{\infty}$, and from (20) we can write $\dot{\tilde{y}} \in l^{\infty}$. Using the Barbalat Lemma [18], since $\tilde{y}(t) \in l^{2}$ and $\dot{\tilde{y}}(t) \in l^{\infty}$ then $\lim _{t \rightarrow \infty} \tilde{y}(t)=0$, which means that $y(t) \rightarrow y(t)^{*}$.

To illustrate the previous result, we will consider the following example. 
Example 3.1

Let us consider the second-order system of form (1) defined as

$$
\begin{aligned}
& \dot{x}(t)=\left[\begin{array}{c}
-3 x_{1}^{2}(t)-x_{2}(t) \\
x_{1}(t)-5 x_{2}^{3}(t)
\end{array}\right]+\left[\begin{array}{l}
1 \\
0
\end{array}\right] u(t) \\
& y(t)=x_{1}(t)
\end{aligned}
$$

where we can identify

$$
f(x)=\left[\begin{array}{c}
-3 x_{1}^{2}-x_{2} \\
x_{1}-5 x_{2}^{3}
\end{array}\right], \quad g(x)=\left[\begin{array}{l}
1 \\
0
\end{array}\right], \quad h(x)=x_{1}
$$

with $x=\left[\begin{array}{ll}x_{1} & x_{2}\end{array}\right]^{\mathrm{T}} \in \mathfrak{R}^{2}$ and $y, u \in \mathfrak{R}$ are scalar functions.

We can readily check that the system has an equilibrium point at $(0,0)$, relative degree $L_{g} h(x)=(\partial h(x) / \partial x) g(x)=1 \neq 0$ and the distribution spanned by $g(x)=\left[\begin{array}{ll}1 & 0\end{array}\right]^{\mathrm{T}}$ is involutive. Since Assumption 2.1 is satisfied, then the system can be represented in form (16) by choosing

$$
\begin{aligned}
y(t) & =x_{1} \in \mathfrak{R}, \quad z(t)=x_{2} \in \mathfrak{R}, \quad \tilde{y}(t)=y(t)-y^{*}(t) \in \mathfrak{R} \\
a(A, y, z) & =-3 y^{2}-z \in \mathfrak{R}, \quad b(B, y, z)=1 \in \mathfrak{R} \\
f_{0}\left(C, y^{*}, z\right) & =-5 z^{3}+y^{*} \in \mathfrak{R}, \quad d(D, y, z)=1 \in \mathfrak{R}
\end{aligned}
$$

It can also be checked that system (25) is locally bounded reference weakly minimum phase according to Definition 3.1. It has associated a positive definite storage function $W_{0}=\frac{1}{2} z^{2}$, with $W_{0}(0)=0$, such that $L_{f_{0}\left(C, y^{*}, z\right)} W_{0}(z)=\left(\partial W_{0}(z) / \partial z\right) f_{0}\left(C, y^{*}, z\right) \leqslant 0[20]$.

Since all the assumptions of Theorem 3.1 are satisfied, then there exists a state feedback of the form (18) with

$$
\begin{aligned}
\omega(t) & =\left[\begin{array}{ll}
-\left(3 y^{2}+z\right) & \left(u^{p}(t)-z+\dot{y}^{*}(t)\right)
\end{array}\right]^{\mathrm{T}} \in \mathfrak{R}^{2} \\
\theta & =\left[\begin{array}{ll}
-1 & 1
\end{array}\right]^{\mathrm{T}} \in \mathfrak{R}^{2}
\end{aligned}
$$

which applied to system (25) makes it locally feedback equivalent to a passive system of the form

$$
\begin{aligned}
& \dot{\tilde{y}}(t)=u^{p}(t)-z(t) \\
& \dot{z}(t)=-5 z^{3}(t)+y^{*}(t)+\tilde{y}(t)
\end{aligned}
$$

with storage function $V(\tilde{y}, z)=\frac{1}{2} \tilde{y}(t)^{2}+W_{0}(z)$, with $W_{0}(z)=\frac{1}{2} z(t)^{2}$. Since the system is locally zero-state observable, applying a controller of the form (19) it is guaranteed that $\lim _{t \rightarrow \infty} \tilde{y}(t)=0$ for any desired bounded trajectory $y^{*}(t) \in \mathfrak{R}$ whose first time derivative is also known. The block diagram of the control scheme for Example 3.1 is shown in Figure 3.

\subsection{Adaptive PBC scheme}

We will consider now the same problem as in Section 3.1 but for the nonlinear system defined as

$$
\begin{aligned}
& \dot{\tilde{y}}(t)=A a^{\prime}\left(\tilde{y}+y^{*}, z\right)+B b^{\prime}\left(\tilde{y}+y^{*}, z\right) u(t)-\dot{y}^{*} \\
& \dot{z}(t)=c\left(C^{\prime}, \tilde{y}+y^{*}, z\right)=f_{0}\left(C, y^{*}, z\right)+d\left(D, \tilde{y}+y^{*}, z\right) \tilde{y}
\end{aligned}
$$




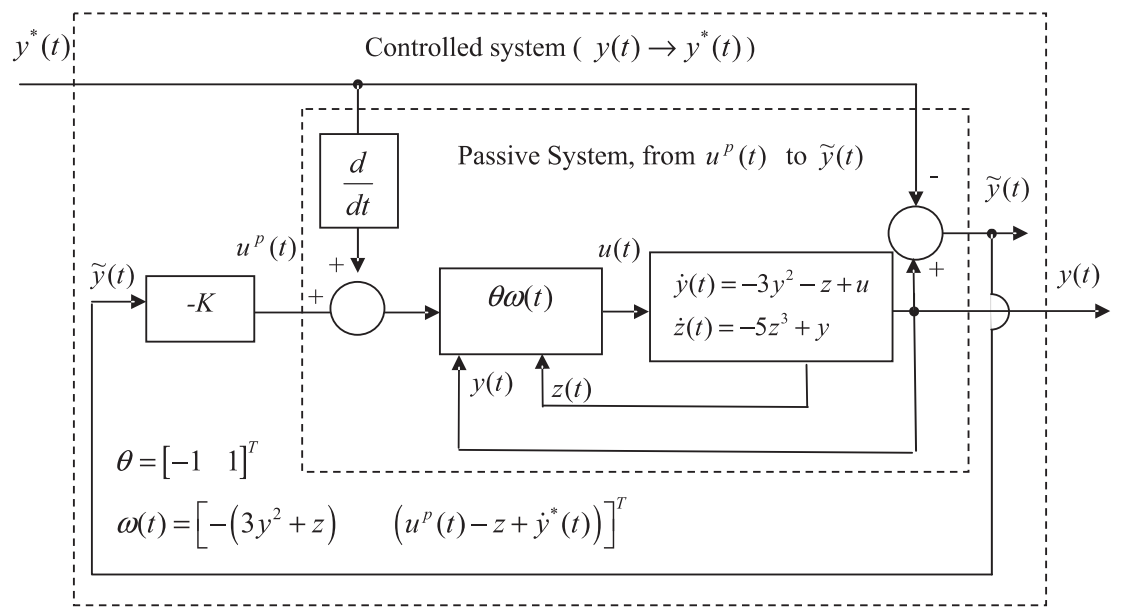

Figure 3. Control scheme for Example 3.1.

which can be written as

$$
\begin{aligned}
& \dot{\tilde{y}}(t)=A a^{\prime}\left(\tilde{y}+y^{*}, z\right)+B b^{\prime}\left(\tilde{y}+y^{*}, z\right) u(t)-\dot{y}^{*} \\
& \dot{z}(t)=f_{0}\left(C, y^{*}, z\right)+d^{\prime}\left(\tilde{y}+y^{*}, z\right) D^{\mathrm{T}} \tilde{y}
\end{aligned}
$$

Here, we have assumed that system parameters have a linear explicit dependence for $a(A, y, z)$, $b(B, y, z)$ and $d(D, y, z)$, and they are unknown. In this case the following theorem can be stated for asymptotic tracking, for the simple case when $B$ is a diagonal matrix. For more general cases see Remark 3.3.

\section{Theorem 3.2}

Let us consider the system defined in (31) and that Assumptions 3.1, 3.2 and 2.4 are satisfied, where $a^{\prime}(y, z) \in \mathfrak{R}^{p}, b^{\prime}(y, z) \in \mathfrak{R}^{m \times m}, f_{0}\left(C, y^{*}, z\right) \in \mathfrak{R}^{n-m}$ and $d^{\prime}(y, z) \in \mathfrak{R}^{(n-m) \times m} . \quad A \in \mathfrak{R}^{m \times p}, B \in$ $\mathfrak{R}^{m \times m}, C \in \mathfrak{R}^{q}$ and $D \in \mathfrak{R}^{m \times m}$ are the unknown system parameters and matrix $B$ is assumed to be diagonal. Then, the following adaptive state feedback

$$
\begin{gathered}
u(t)=b^{\prime}(y, z)^{-1} \theta(t) \omega(t) \\
\theta(t)=\left[\begin{array}{lll}
\theta_{1}(t) & \theta_{2}(t) & \theta_{3}(t)
\end{array}\right] \in \mathfrak{R}^{m \times(p+2 m)} \quad \text { with } \theta_{1}(t) \in \mathfrak{R}^{m \times p}, \theta_{2}(t) \in \mathfrak{R}^{m \times m}, \theta_{3}(t) \in \mathfrak{R}^{m \times m} \\
\omega(t)=\left[\begin{array}{lll}
a^{\prime}(y, z)^{\mathrm{T}} & \left(L_{d^{\prime}\left(\tilde{y}+y^{*}, z\right)} W_{0}(z)\right)^{\mathrm{T}} & \left(u^{p}(t)+\dot{y}^{*}(t)\right)^{\mathrm{T}}
\end{array}\right]^{\mathrm{T}} \in \mathfrak{R}^{(p+2 m)}
\end{gathered}
$$

with the adaptive law

$$
\dot{\theta}(t)=-\operatorname{sign}(B) \tilde{y}(t) \omega^{\mathrm{T}}(t)
$$

makes system (31) equivalent to a passive one with a $C^{2}$ storage function. $\operatorname{sign}(B)$ represents a diagonal matrix in whose diagonal are located the sign of the elements of the diagonal matrix $B$, which are assumed to be known. Furthermore, if we suppose that the system is locally 
zero- state observable, then applying the controller

$$
u^{p}(t)=-K \tilde{y}(t)
$$

with $K \in \mathfrak{R}^{m \times m}$ any positive definite matrix and $\tilde{y}(t)=y(t)-y(t)^{*} \in \mathfrak{R}^{m}$, the resulting overall system has bounded trajectories and $\lim _{t \rightarrow \infty} \tilde{y}(t)=y(t)-y(t)^{*}=0$.

Proof

After applying the state feedback (32) to system (31) we can obtain the following:

$$
\begin{gathered}
\dot{\tilde{y}}(t)=A a^{\prime}\left(\tilde{y}+y^{*}, z\right)+B \theta(t) \omega(t) \\
\dot{z}(t)=f_{0}\left(C, \tilde{y}+y^{*}, z\right)+d^{\prime}\left(\tilde{y}+y^{*}, z\right) D^{\mathrm{T}} \tilde{y}
\end{gathered}
$$

which considering the definition of $\theta(t)$ and $\omega(t)$ given in (32) is equal to

$$
\begin{aligned}
& \dot{\tilde{y}}(t)=A a^{\prime}\left(\tilde{y}+y^{*}, z\right)+B \theta_{1} a^{\prime}\left(\tilde{y}+y^{*}, z\right)+B \theta_{2} L_{d^{\prime}\left(\tilde{y}+y^{*}, z\right)} W_{0}(z)+B \theta_{3} u^{p}+B \theta_{3} \dot{y}^{*}-\dot{y}^{*} \\
& \dot{z}(t)=f_{0}\left(C, y^{*}, z\right)+d^{\prime}\left(\tilde{y}+y^{*}, z\right) D^{\mathrm{T}} \tilde{y}
\end{aligned}
$$

Adding and subtracting the term $u^{p}(t)$, the previous expression can be written as

$$
\begin{aligned}
& \dot{\tilde{y}}(t)=\left(A+B \theta_{1}\right) a^{\prime}\left(\tilde{y}+y^{*}, z\right)+B \theta_{2} L_{d^{\prime}\left(\tilde{y}+y^{*}, z\right)} W_{0}(z)+\left(B \theta_{3}-I\right) u^{p}+\left(B \theta_{3}-I\right) \dot{y}^{*}+u^{p} \\
& \dot{z}(t)=f_{0}\left(C, y^{*}, z\right)+d^{\prime}\left(\tilde{y}+y^{*}, z\right) D^{\mathrm{T}} \tilde{y}
\end{aligned}
$$

We define the ideal passivator parameters as

$$
\begin{gathered}
A+B \theta_{1}^{*}=I \Rightarrow \theta_{1}^{*}=-B^{-1} A \quad \text { with } \theta_{1}^{*} \in \mathfrak{R}^{m \times p} \\
D+B \theta_{2}^{*}=I \Rightarrow \theta_{2}^{*}=-B^{-1} D \quad \text { with } \theta_{2}^{*} \in \mathfrak{R}^{m \times m} \\
B \theta_{3}^{*}-I=0 \Rightarrow \theta_{3}^{*}=B^{-1} \quad \text { with } \theta_{3}^{*} \in \mathfrak{R}^{m \times m}
\end{gathered}
$$

Then we can define the parameter error matrix as

$$
\Phi(t)=\theta(t)-\theta^{*}=\left[\begin{array}{lll}
\Phi_{1}(t) & \Phi_{2}(t) & \Phi_{3}(t)
\end{array}\right] \in \mathfrak{R}^{m \times(p+2 m)}
$$

with

$$
\begin{aligned}
\theta(t) & =\left[\begin{array}{lll}
\theta_{1}(t) & \theta_{2}(t) & \theta_{3}(t)
\end{array}\right] \in \mathfrak{R}^{m \times(p+2 m)} \\
\theta^{*} & =\left[\begin{array}{lll}
\theta_{1}^{*} & \theta_{2}^{*} & \theta_{3}^{*}
\end{array}\right] \in \mathfrak{R}^{m \times(p+2 m)}
\end{aligned}
$$

Let us consider the following storage function:

$$
V(\tilde{y}, z, \Phi)=\frac{1}{2} \tilde{y}^{\mathrm{T}}(t) \tilde{y}(t)+W_{0}(z)+\frac{1}{2} \operatorname{Trace}\left(|B| \Phi(t)^{\mathrm{T}} \Phi(t)\right)
$$

where $|B|$ represents a diagonal matrix in whose diagonal are located the absolute values of the diagonal elements of matrix $B$, which are assumed to be unknown. The first time derivative of $V$ is equal to

$$
\dot{V}(\tilde{y}, z, \Phi)=\tilde{y}(t)^{\mathrm{T}} \dot{\tilde{y}}(t)+\dot{W}_{0}+\operatorname{Trace}\left(|B| \dot{\Phi}(t)^{\mathrm{T}} \Phi(t)\right)
$$

Substituting $\dot{\tilde{y}}(t)$ from Equation (36) in the previous expression and regrouping we obtain

$$
\begin{aligned}
\dot{V}(\tilde{y}, z, \Phi)= & \tilde{y}^{\mathrm{T}}(t)\left(A+B \theta_{1}\right) a^{\prime}\left(\tilde{y}+y^{*}, z\right)+\tilde{y}^{\mathrm{T}}(t) B \theta_{2} L_{d^{\prime}\left(\tilde{y}+y^{*}, z\right)} W_{0}(z)+\tilde{y}^{\mathrm{T}}(t)\left(B \theta_{3}-I\right) u^{p} \\
& +\tilde{y}^{\mathrm{T}}(t)\left(B \theta_{3}-I\right) \dot{y}^{*}+\tilde{y}^{\mathrm{T}}(t) u^{p}+\frac{\partial W_{0}(z)}{\partial z} \dot{z}(t)+\operatorname{Trace}\left(|B| \dot{\Phi}(t)^{\mathrm{T}} \Phi(t)\right)
\end{aligned}
$$


Replacing $\dot{z}(t)$ from (36) and using definitions (37) we get

$$
\begin{aligned}
\dot{V}(\tilde{y}, z, \Phi)= & \tilde{y}^{\mathrm{T}}(t) u^{p}(t)+\tilde{y}^{\mathrm{T}}(t)\left(B \theta_{1}-B \theta_{1}^{*}\right) a^{\prime}\left(\tilde{y}+y^{*}, z\right)+\tilde{y}^{\mathrm{T}}(t) B \theta_{2} L_{d^{\prime}}\left(\tilde{y}+y^{*}, z\right) \\
& +\tilde{y}^{\mathrm{T}}(t)\left(B \theta_{3}-B \theta_{3}^{*}\right) u^{p}(t)+\left(B \theta_{3}-B \theta_{3}^{*}\right) \dot{y}^{*}(t)+\left(\frac{\partial W_{0}(z)}{\partial z}\right)^{\mathrm{T}} f_{0}\left(C, y^{*}, z\right) \\
& +\left(\frac{\partial W_{0}(z)}{\partial z}\right)^{\mathrm{T}} d\left(\tilde{y}+y^{*}, z\right) D^{\mathrm{T}} \tilde{y}(t)+\operatorname{Trace}\left(|B| \dot{\Phi}(t)^{\mathrm{T}} \Phi(t)\right)
\end{aligned}
$$

or equivalently

$$
\begin{aligned}
\dot{V}(\tilde{y}, z, \Phi)= & \tilde{y}^{\mathrm{T}}(t) u^{p}(t)+L_{f_{0}\left(C, y^{*}, z\right)} W_{0}(z)+\tilde{y}^{\mathrm{T}}(t) B\left(\theta_{1}-\theta_{1}^{*}\right) a^{\prime}\left(\tilde{y}+y^{*}, z\right) \\
& +\tilde{y}^{\mathrm{T}}(t) B \theta_{2} L_{d^{\prime}\left(\tilde{y}+y^{*}, z\right)} W_{0}(z)+\tilde{y}^{\mathrm{T}}(t) B\left(\theta_{3}-\theta_{3}^{*}\right) u^{p}+B\left(\theta_{3}-\theta_{3}^{*}\right) \dot{y}^{*}(t) \\
& +\left(L_{d^{\prime}\left(\tilde{y}+y^{*}, z\right)} W_{0}(z)\right)^{\mathrm{T}} D^{\mathrm{T}} \tilde{y}(t)+\operatorname{Trace}\left(|B| \dot{\Phi}(t)^{\mathrm{T}} \Phi(t)\right)
\end{aligned}
$$

Regrouping terms we obtain

$$
\begin{aligned}
\dot{V}(\tilde{y}, z, \Phi)= & \tilde{y}^{\mathrm{T}}(t) u^{p}(t)+L_{f_{0}\left(C, y^{*}, z\right)} W_{0}(z)+\tilde{y}^{\mathrm{T}}(t) B \Phi_{1} a^{\prime}\left(\tilde{y}+y^{*}, z\right)+\tilde{y}^{\mathrm{T}}(t)\left(D+B \theta_{2}\right) L_{d^{\prime}\left(\tilde{y}+y^{*}, z\right)} W_{0}(z) \\
& +\tilde{y}^{\mathrm{T}}(t) B \Phi_{3}\left(u^{p}(t)+\dot{y}^{*}(t)\right)+\operatorname{Trace}\left(|B| \dot{\Phi}(t)^{\mathrm{T}} \Phi(t)\right)
\end{aligned}
$$

Using the definition of $\theta_{2}^{*}$ given by (37) we have the following:

$$
\begin{aligned}
\dot{V}(\tilde{y}, z, \Phi)= & \tilde{y}^{\mathrm{T}}(t) u^{p}(t)+L_{f_{0}\left(C, y^{*}, z\right)} W_{0}(z)+\tilde{y}^{\mathrm{T}}(t) B \Phi_{1} a^{\prime}\left(\tilde{y}+y^{*}, z\right) \\
& +\tilde{y}^{\mathrm{T}}(t)\left(B \theta_{2}-B \theta_{2}^{*}\right) L_{d^{\prime}\left(\tilde{y}+y^{*}, z\right)} W_{0}(z) \\
& +\tilde{y}^{\mathrm{T}}(t) B \Phi_{3}\left(u^{p}(t)+\dot{y}^{*}(t)\right)+\operatorname{Trace}\left(|B| \dot{\Phi}(t)^{\mathrm{T}} \Phi(t)\right)
\end{aligned}
$$

In a more compact form we write

$$
\dot{V}(\tilde{y}, z, \Phi)=\tilde{y}^{\mathrm{T}}(t) u^{p}(t)+L_{f_{0}\left(C, y^{*}, z\right)} W_{0}(z)+\tilde{y}^{\mathrm{T}}(t) B \Phi(t) \omega(t)+\operatorname{Trace}\left(|B| \dot{\Phi}(t)^{\mathrm{T}} \Phi(t)\right)
$$

Using the property of two vectors that $a^{\mathrm{T}} b=b^{\mathrm{T}} a=\operatorname{Trace}\left(b a^{\mathrm{T}}\right)=\operatorname{Trace}\left(a b^{\mathrm{T}}\right)$ and regrouping terms we get

$$
\dot{V}(\tilde{y}, z, \Phi)=\tilde{y}^{\mathrm{T}}(t) u^{p}(t)+L_{f_{0}\left(C, y^{*}, z\right)} W_{0}(z)+\operatorname{Trace}\left\{\left[B^{\mathrm{T}} \tilde{y}(t) \omega(t)^{\mathrm{T}}+|B| \dot{\Phi}(t)^{\mathrm{T}}\right]\right\} \Phi(t)
$$

Substituting now the adaptive law defined in Equation (33), expressed as $\dot{\Phi}(t)=\dot{\theta}(t)=$ $-\operatorname{sign}(B) \tilde{y}(t) \omega^{\mathrm{T}}(t)$ and considering $B^{\mathrm{T}}=B=|B| \operatorname{sign}(B)$, we finally obtain the following:

$$
\dot{V}(\tilde{y}, z, \Phi)=\tilde{y}^{\mathrm{T}}(t) u^{p}(t)+L_{f_{0}\left(C, y^{*}, z\right)} W_{0}(z)
$$

Using Assumption 3.2 we can finally write

$$
\dot{V}(\tilde{y}, z, \Phi) \leqslant \tilde{y}^{\mathrm{T}}(t) u^{p}(t)
$$

Thus, from Definition 2.1, the resultant system is passive from $u^{p}(t)$ to $\tilde{y}(t)$. Following the same reasoning as in Theorem 3.1 and considering again the controller $u^{p}(t)=-K \tilde{y}(t)$, together with Assumptions 3.1 and 3.2 , we can assure that trajectories of the adaptive system are bounded 
and also we can guarantee that $\lim _{t \rightarrow \infty} \tilde{y}(t)=0$, provided the system is locally zero-state observable.

\section{Remark 3.1}

In the previous development, unity adaptive gains were used. It can be shown [14-17] that the same results hold if, positive scalar, positive definite constant matrix and positive definite timevarying matrix adaptive gains are introduced.

\section{Remark 3.2}

In the proposed scheme, parametric convergence to the ideal controller parameters is not guaranteed. This is only achieved if persistently exciting conditions of some signal vectors are satisfied [14-17].

\section{Remark 3.3}

For easy exposition the results of Theorem 3.2 were derived assuming that $B$ is a diagonal matrix. However, it is possible to show that the same results can be achieved for the cases when $B$ is a positive definite matrix and when $B$ is a general matrix [14-17].

To illustrate the previous result we will apply the results of Theorem 3.2 to the same system used in Example 3.1.

\section{Example 3.2}

Let us control the same system of Example 3.1 but this time we will assume that the parameters are unknown. Since system (25) satisfies Assumption 2.1 it can be expressed in form (31) as follows:

$$
\begin{aligned}
& \dot{\tilde{y}}(t)=\left[\begin{array}{ll}
-3 & -1
\end{array}\right]\left[\begin{array}{c}
y^{2}(t) \\
z(t)
\end{array}\right]+u(t)-\dot{y}^{*}(t) \\
& \dot{z}(t)=-5 z^{3}(t)+y^{*}(t)+\tilde{y}(t)
\end{aligned}
$$

by choosing

$$
\begin{aligned}
y(t) & =x_{1} \in \mathfrak{R}, \quad z(t)=x_{2} \in \mathfrak{R}, \quad \tilde{y}(t)=y(t)-y^{*}(t) \in \mathfrak{R} \\
A & =\left[\begin{array}{ll}
-3 & -1
\end{array}\right] \in \mathfrak{R}^{2}, \quad a^{\prime}(y, z)=\left[\begin{array}{c}
y^{2} \\
z
\end{array}\right] \in \mathfrak{R}^{2} \\
B & =1 \in \mathfrak{R}, \quad b^{\prime}(y, z)=1 \in \mathfrak{R} \\
f_{0}\left(C, y^{*}, z\right) & =-5 z^{3}+y^{*} \in \mathfrak{R}, \quad d^{\prime}(y, z)=1 \in \mathfrak{R}, \quad D=1 \in \mathfrak{R}
\end{aligned}
$$

Parameters $A=\left[\begin{array}{ll}-3-1 & -1\end{array} \in \mathfrak{R}^{2}, B=1 \in \mathfrak{R}\right.$ and $D=1 \in \mathfrak{R}$ are assumed to be unknown. The state feedback (27) is now defined as

$$
u(t)=b^{\prime}(y, z)^{-1} \theta^{\mathrm{T}}(t) \omega(t)
$$

with $\theta(t)=\left[\begin{array}{lll}\theta_{1}^{\mathrm{T}}(t) & \theta_{2}(t) & \theta_{3}(t)\end{array}\right]^{\mathrm{T}} \in \mathfrak{R}^{4}, \theta_{1}(t)=\left[\theta_{1}^{1}(t) \theta_{1}^{2}(t)\right]^{\mathrm{T}} \in \mathfrak{R}^{2}, \theta_{2}(t) \in \mathfrak{R}, \theta_{3}(t) \in \mathfrak{R}$ and $\omega(t)=$

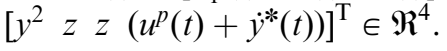




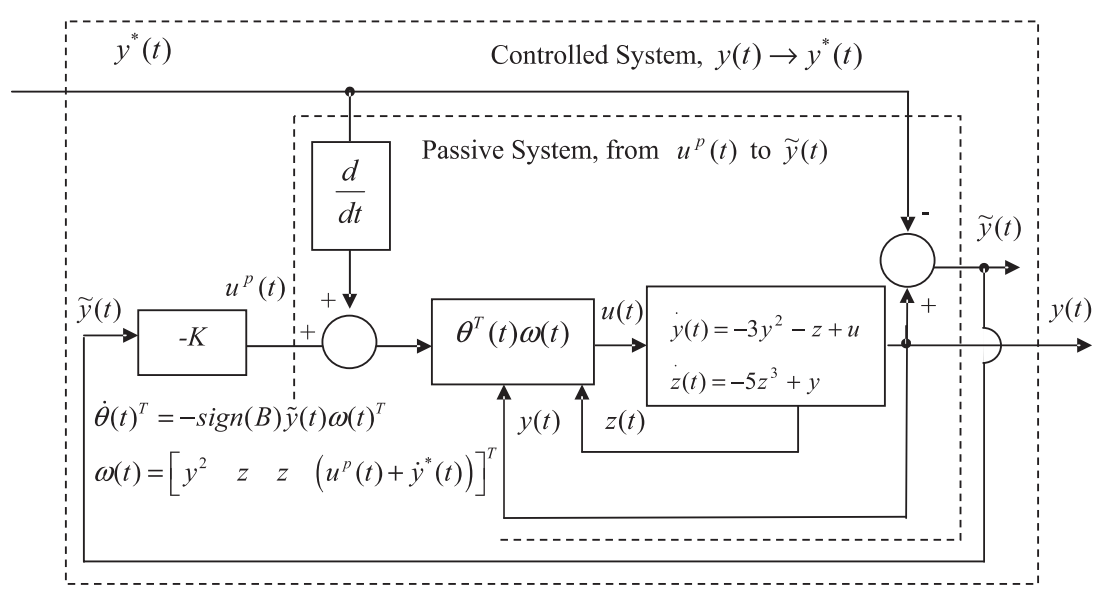

Figure 4. Adaptive control scheme for Example 3.2.

The ideal controller parameters are defined as $\theta_{1}^{1^{*}}=3, \theta_{1}^{2^{*}}=1, \theta_{2}^{*}=-1$ and $\theta_{3}^{*}=1$. If we apply the state feedback (42) to system (41) we will have the following:

$$
\begin{aligned}
& \dot{\tilde{y}}(t)=-\tilde{y}^{\mathrm{T}}(t) u^{p}(t)+B \phi(t) \omega(t) \\
& \dot{z}(t)=-5 z^{3}+y^{*}+\tilde{y}
\end{aligned}
$$

where $\phi(t)=\theta(t)-\theta^{*}=\left[\begin{array}{llll}\theta_{1}^{1}(t) & \theta_{1}^{2}(t) & \theta_{2}(t) & \theta_{3}(t)\end{array}\right]^{\mathrm{T}}-\left[\begin{array}{llll}\theta_{1}^{1 *} & \theta_{1}^{2 *} & \theta_{2}^{*} & \theta_{3}^{*}\end{array}\right]^{\mathrm{T}} \in \mathfrak{R}^{4}$. The adaptive law to adjust the state feedback parameter is given by (33) and has the form

$$
\dot{\theta}(t)=-\tilde{y}(t)\left[y^{2} \quad z \quad z \quad\left(u^{p}(t)+\dot{y}^{*}(t)\right)\right]^{\mathrm{T}}
$$

This guarantees that system (43) is passive from $u^{p}(t)$ to $\tilde{y}(t)$, with storage function $V(\tilde{y}, z, \phi)=\frac{1}{2} \tilde{y}^{2}(t)+W_{0}(z)+\frac{1}{2}|B| \phi(t)^{\mathrm{T}} \phi(t)$ and $W_{0}(z)=\frac{1}{2} z^{2}(t)$. Furthermore, if we choose $u^{p}(t)$ as in (34), then $\lim _{t \rightarrow \infty} \tilde{y}(t)=0$ for all bounded desired trajectory $y^{*}(t) \in \mathfrak{R}$ with bounded continuous first time derivative, while all the signals remain bounded. The block diagram of the control scheme is shown in Figure 4.

\section{SIMULATION RESULTS AND TRANSIENT BEHAVIOUR}

In this section we present the simulation results corresponding to Examples 3.1 and 3.2. All the simulations were done in Matlab 6.5 using variable-step, ode45 (Dorman-Prince) method for solving the differential equations with relative and absolute tolerance of $10^{-6}$.

We first consider the system of Example 3.1 with $u(t)=0$, to study its natural behaviour for further comparison purposes. Figure 5 shows the evolution of the state of the system when no control is applied and initial conditions $y(0)=0$ and $z(0)=-0.25$ are selected.

It can be seen that the system exhibits oscillations and a stable internal dynamic $z(t)$.

Two different reference signals will be applied to this system, as shown in Figures 6(a) and (b). The first reference signal is used to study the behaviour of the system under tracking control, 
and the second one is for regulation purposes. In Section 4.1, the simulation results of the control schemes applied to the system of Example 3.1 are presented and in Section 4.2 the corresponding results for the system of Example 3.2 are discussed.
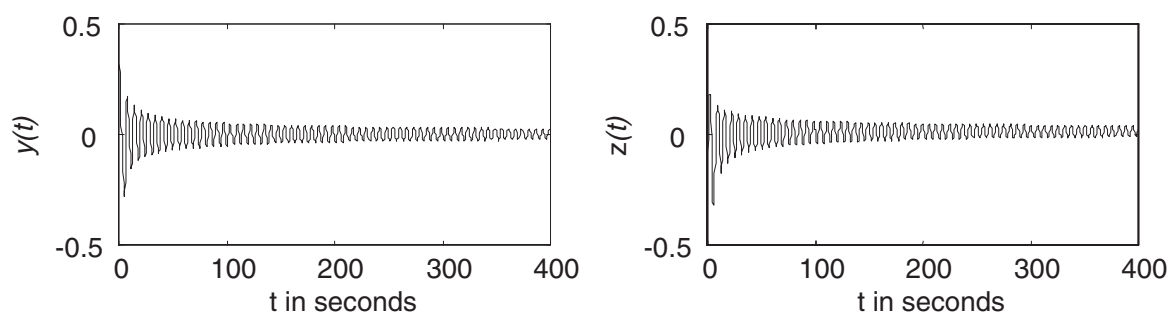

Figure 5. Natural behaviour of the system of Example 3.1.
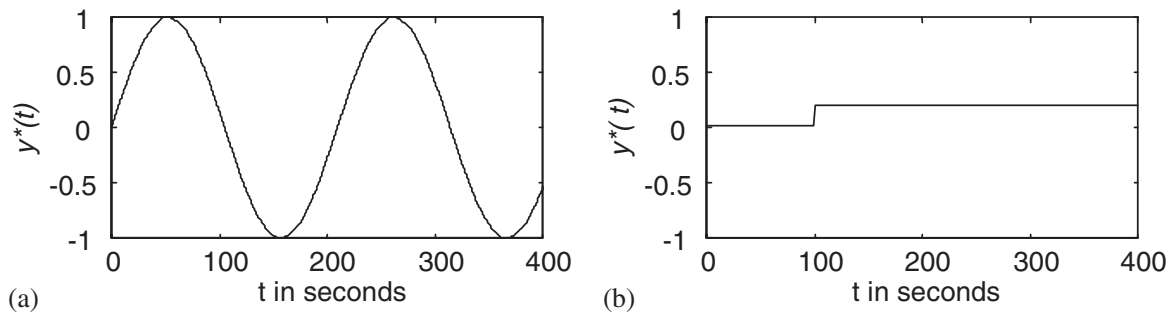

Figure 6. Reference signals applied to the system of Example 3.1 and Example 3.2: (a) sine wave of amplitude 1 and frequency $0.03 \mathrm{rad} / \mathrm{s}$; and (b) step signal of amplitude 0.1 applied at $t=100$.
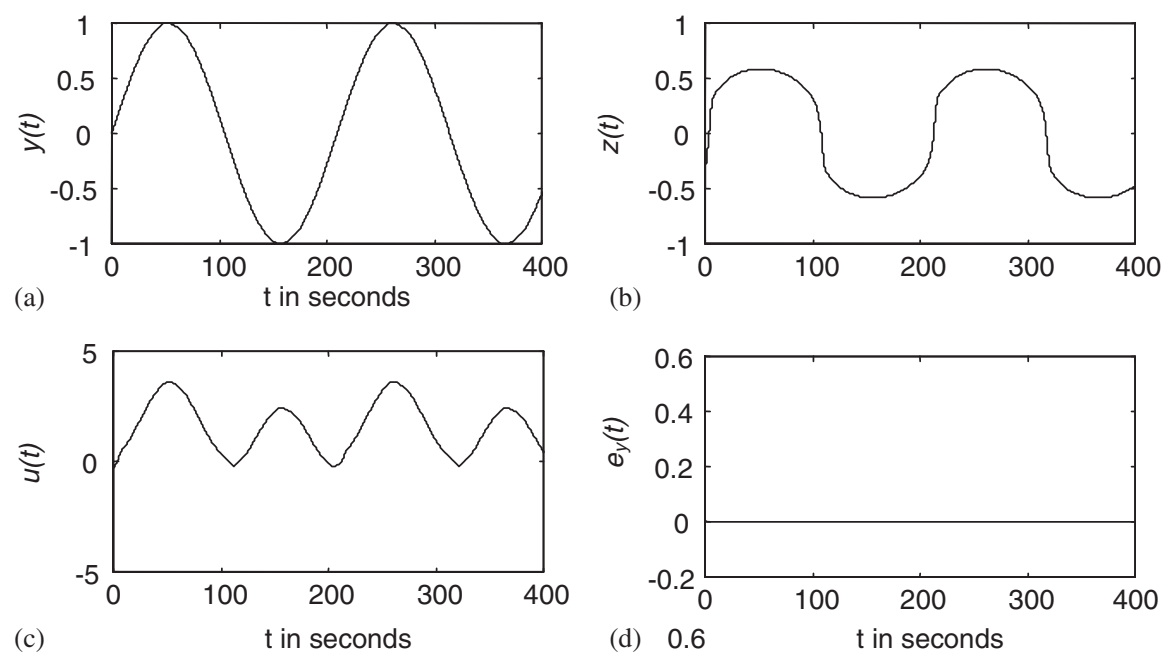

Figure 7. Simulation results for tracking control scheme of Example 3.1. Non-adaptive case. 


\subsection{Simulation results for Example 3.1}

The evolution of the state, as well as the control input applied to the plant of Example 3.1 and the tracking error, are shown in Figure 7 when a feedback gain $K=-40$ is chosen and a sinusoidal reference signal like the one shown in Figure 6(a) is considered. The initial conditions were chosen as $y(0)=0$ and $z(0)=-0.25$.

It is observed from Figure 7(d) that the tracking error is zero for all $t$. This is due to the exact cancellation, and the zero initial condition of the output $y(t)$. The same results are presented in Figure 8 but for the case when the reference signal is the constant shown in Figure 6(b).
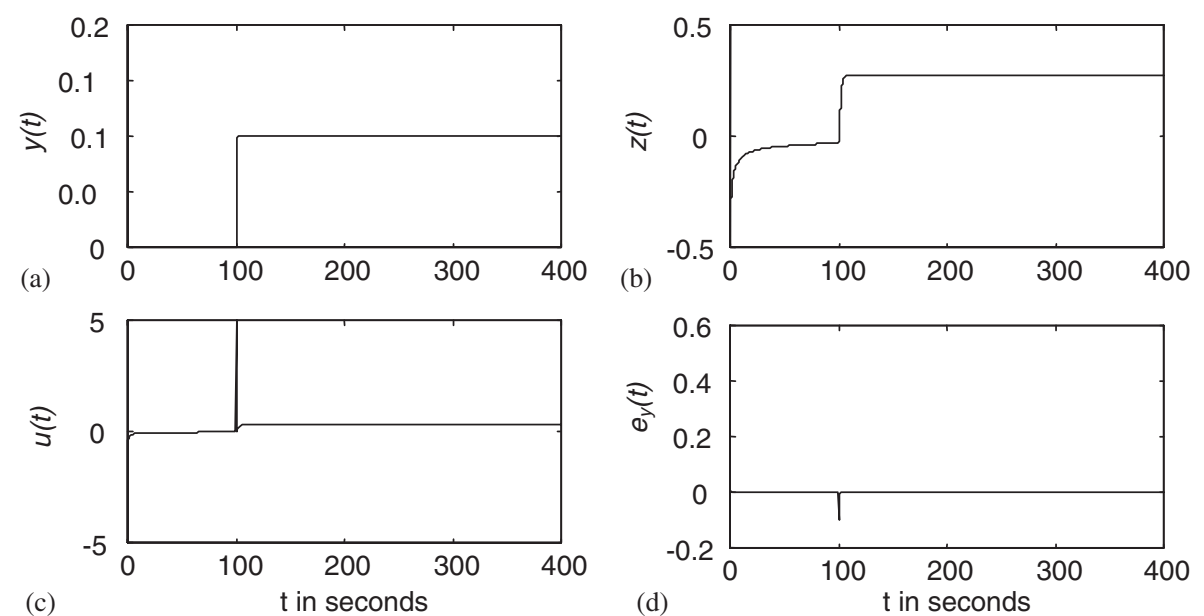

Figure 8. Simulation results for the regulation control scheme of Example 3.1. Non-adaptive case.
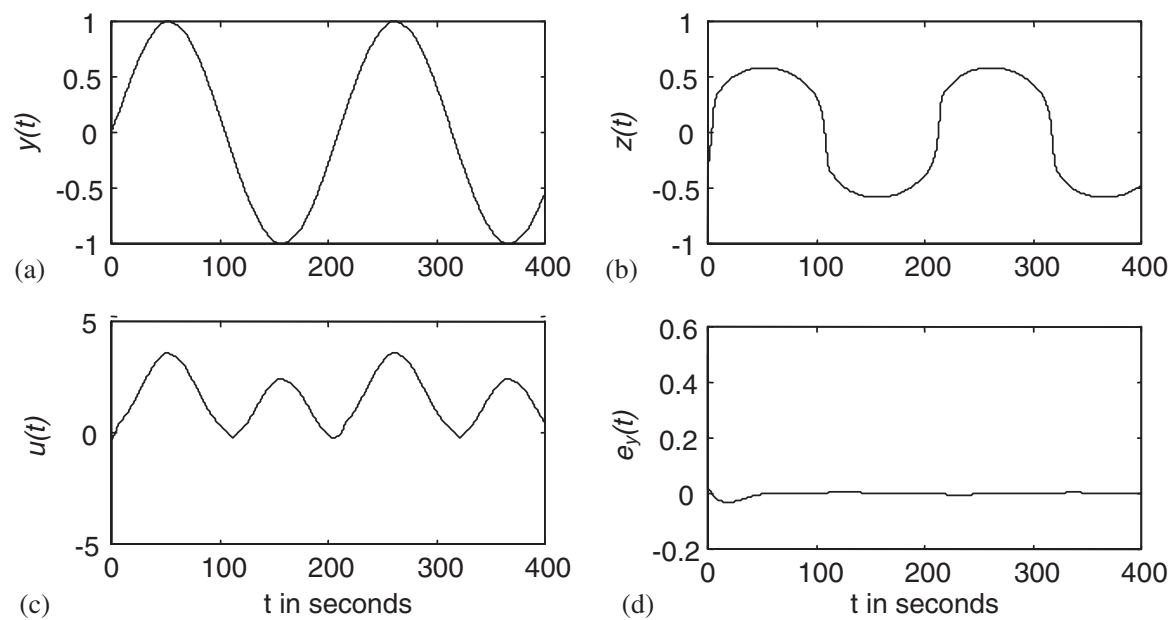

Figure 9. Simulation results for the tracking adaptive control scheme of Example 3.2. Evolution of the main variables. 
A similar behaviour to that exhibited in the previous case is observed here under regulation. The tracking error is zero except at $t=100$. It is important to note that when applying a step reference signal at $t=100$, its derivative is undefined at the moment the step is applied. So in this case a practical solution could be implemented using a saturation block.

\subsection{Simulation results for Example 3.2}

We now consider the system defined in Example 3.2, with the assumption that the parameters are unknown. In Figure 9 the evolution of the state, the control input and the tracking error, is shown when the sinusoidal reference signal depicted in Figure 6(a) is applied, and a feedback gain $K=-40$ was selected. The initial conditions were chosen again as $y(0)=0$ and $z(0)=-0.25$.

As expected, the tracking error shown in Figure 9(d) goes to zero as $t$ goes to infinity so the output of the plant asymptotically tracks the reference signal. This adaptive control scheme is a bit slower than the non-adaptive scheme. In Figure 10 the evolution of the state feedback parameters $\theta(t)$ can be seen when the following initial conditions were chosen $\theta_{1}^{1}(0)=0$, $\theta_{1}^{2}(0)=0.5, \theta_{2}(0)=0$ and $\theta_{3}(0)=0$.

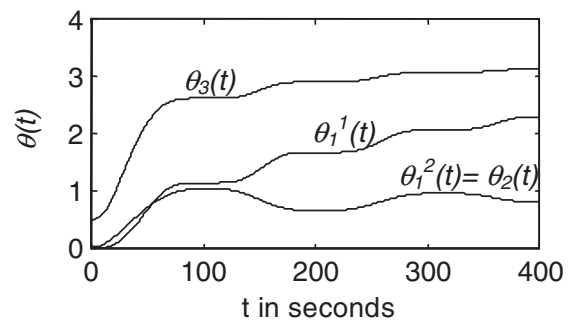

Figure 10. Simulation results for the tracking adaptive control scheme of Example 3.2.

Evolution of the state feedback parameter.
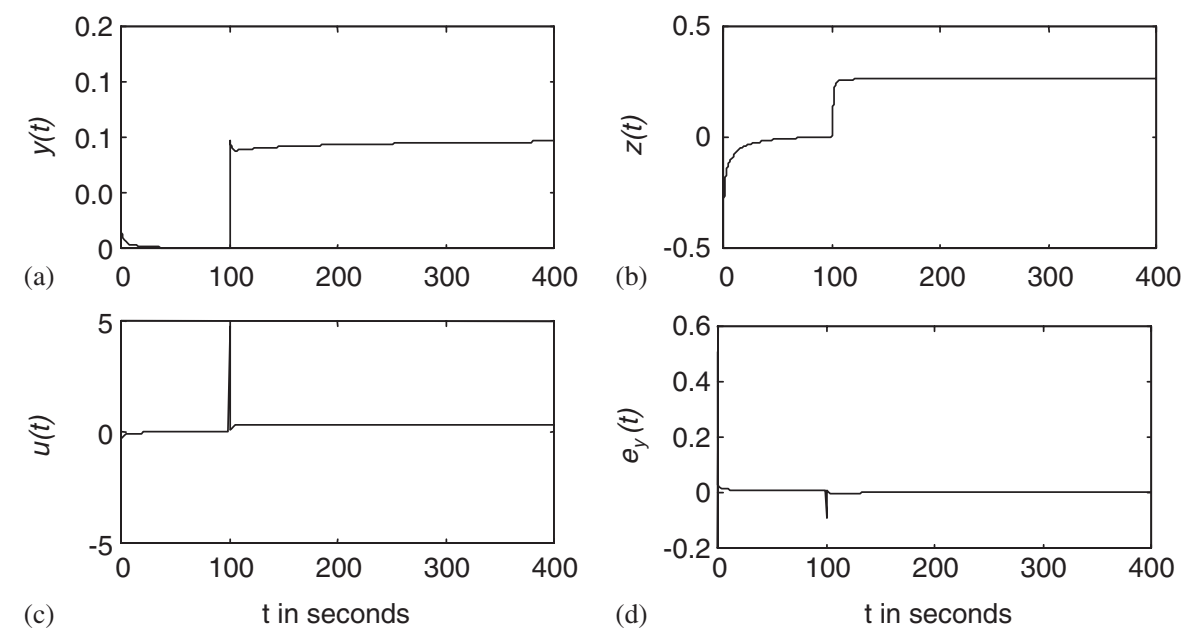

Figure 11. Simulation results for the adaptive regulation control scheme of Example 3.2. Evolution of the main variables. 


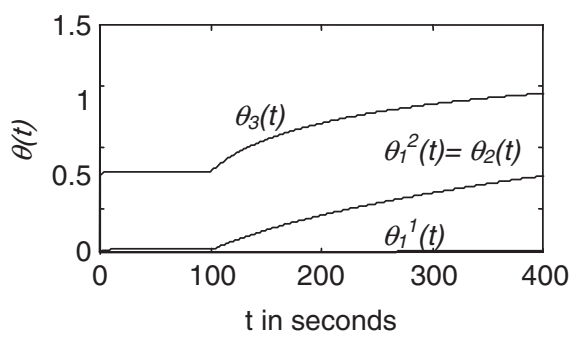

Figure 12. Simulation results for the adaptive regulation control scheme of Example 3.2. Evolution of the state feedback parameters.

As it is shown in [19] the state feedback parameter $\theta(t)$ is adjusted in such a way that zero control error is guaranteed, minimizing at the same time an energy function. For this adaptive case, the transient behaviour depends on the adaptive law used and on the adaptive gains that one introduces. Different adaptive laws can be used, depending on the control purpose [19].

The same study as in the previous case was done using a feedback gain $K=-40$, when the constant reference signal depicted in Figure 6(b) is applied. In Figure 11 it is shown the evolution of the state, the control input and the tracking error, observing from Figure 11(d) that the tracking error is driven to zero, with a jump at the instant when the step is applied. The parameters' evolution is shown in Figure 12.

\section{CONCLUSIONS}

In this paper, a new passivity-based control (PBC) scheme, which considers a controller and a state feedback, is proposed in order to solve tracking, regulation and stabilization problems for a class of nonlinear system. Besides, two different state feedbacks are proposed which work properly with a simple proportional controller. A non-adaptive state feedback is presented when all system parameters are known, while the adaptive state feedback is for the case when system parameters are unknown. The class of nonlinear systems studied corresponds to time-invariant MIMO systems with relative degree 1 and locally bounded reference weakly minimum phase. The resultant scheme guarantees that the overall system is stable (all the signals remain bounded) and $\lim _{t \rightarrow \infty} \tilde{y}(t)=y(t)-y(t)^{*}=0$, where $y(t)^{*}$ is a bounded arbitrary trajectory. Two examples were studied under simulations to verify the theoretical results, considering first the case when all parameters are known, and then the case of unknown parameters. All the simulation results are in complete agreement with the theoretical results presented in Sections 3 and 4.

\section{ACKNOWLEDGEMENTS}

The results reported in this paper have been supported by CONICYT-CHILE through grant FONDECYT No. 1030962. We are very grateful for the valuable observations and suggestions made by the reviewers to improve the final version of this paper. 


\section{REFERENCES}

1. Aeyels D. Stabilization of a class of nonlinear systems by a smooth feedback control. Systems and Control Letters 1985; 5:289-294.

2. Hill D, Moylan P. Stability results for nonlinear feedback systems. Automatica 1977; 13:377-382.

3. Willems JC. Dissipative dynamical systems Part I: general theory. Archive for Rational Mechanics and Analysis 1972; 45:321-351.

4. Willems JC. Dissipative dynamical systems Part II: linear systems with quadratic supply rates. Archive for Rational Mechanics and Analysis 1972; 45:352-393.

5. Byrnes CI, Isidori A, Willems JC. Passivity, feedback equivalence, and the global stabilization of minimum phase nonlinear systems. IEEE Transactions on Automatic Control 1991; 36(11):1228-1240.

6. Marino R, Tomei P. Robust stabilization of feedback linearizable time-varying uncertain nonlinear systems. Automatica 1993; 29(1):181-189.

7. Lin W, Shen T. Robust passivity feedback design for minimum-phase nonlinear systems with structural uncertainty. Automatica 1999; 35(1):35-47.

8. Seron MM, Hill DJ, Fradkov AL. Nonlinear adaptive control of feedback passivity systems. Automatica 1995; 31:1053-1060.

9. Su W, Xie L. Robust control of nonlinear feedback passive systems. Systems and Control Letters 1996; 28:85-93.

10. Karagiannis D, Astolfi A, Ortega R. Two results for adaptive output feedback stabilization of nonlinear systems. Automatica 2003; 39(5):857-866.

11. Hsiao FH, Pan ST, Fann SL. Stabilization of a class of nonlinear control systems via dynamic output feedback. The Journal of the Franklin Institute 1995; 332B(3):263-273.

12. Teel AR, Praly L. Global stabilizability and observability imply semi-global stabilizability by output feedback. Systems and Control Letters 1994; 22:313-325.

13. Choi HL, Lim JT. Global exponential stabilization of a class of nonlinear systems by output feedback. IEEE Transactions on Automatic Control 2005; 50(2):255-257.

14. Duarte-Mermoud MA, Castro-Linares R, Castillo-Facuse A. Adaptive passivity of nonlinear systems using timevarying gains. Dynamics and Control 2001; 11(4):333-351.

15. Duarte-Mermoud MA, Castro-Linares R, Castillo-Facuse A. Direct passivity of a class of MIMO nonlinear systems using adaptive feedback. International Journal of Control 2002; 75(1):23-33.

16. Duarte-Mermoud MA, Méndez-Miquel JM, Castro-Linares R, Castillo-Facuse A. Adaptive paésivation with timevarying gains of MIMO nonlinear systems. Kybernetes 2003; 32(9/10):1342-1368.

17. Castro-Linares R, Duarte-Mermoud MA. Passivity equivalence of a class of nonlinear systems via adaptive feedback. Proceedings of the 8th Latinamerican Congress on Automatic Control, Marbella, Chile. ACCA: Santiago, Chile, 9-13 November 1998; 249-254.

18. Narendra KS, Annaswamy AM. Stable Adaptive Systems. Prentice-Hall: Englewood Cliffs, NJ, 1989.

19. Travieso-Torres JC, Duarte-Mermoud MA. Equivalence between adaptive passivity based control and model reference adaptive control. WSEAS Transactions on Circuits and Systems 2004; 4(9):1912-1917.

20. Khalil HK. Nonlinear Systems (3rd edn). Prentice-Hall: Englewood Cliffs, NJ, 2002. 